\title{
Deep Vein Thrombosis: A Rare Association with Tuberculosis
}

\author{
SUPRIYA SARKAR, ${ }^{1}$ KAUSHIK SAHA, ${ }^{2}$ MALAY KUMAR MAIKAP, ${ }^{3}$ DEBRAJ JASH ${ }^{4}$
}

\begin{abstract}
:
We are reporting two cases of bilateral extensive lower leg deep vein thrombosis in young male patients without any risk factors. First case was diagnosed as left sided tuberculous pleural effusion and second case was sputum smear positive pulmonary tuberculosis. In both patients, deep vein thrombosis developed during first month of treatment and inguinal pain was the first symptom. The second case had an attack of pulmonary thromboembolism. The cause effect relationship between deep vein thrombosis and tuberculosis or anti-tubercular drug (particularly rifampicin) has been discussed as such association may have significant therapeutic implications.
\end{abstract}

Keyword: Tuberculosis, lower limb, deep vein thrombosis, anti-tuberculosis drugs, rifampicin.

\section{Introduction:}

Tubercular pleural effusion, occurs as a result of hypersensitivity reaction to tubercular protein, is usual have an uncomplicated course. Tubercular empyema, occurs as a result of rupture of underlying tubercular cavity, can have a wide variety of local complications, and most of them are not life threatening. Pleural tuberculosis (Pl-TB) as well as pulmonary tuberculosis (P-TB) is usually not associated with hematological complication. There are few reports of systemic hematological complications, like disseminated intra-vascular coagulation (DIC) and deep vein thrombosis (DVT), associated with P-TB. ${ }^{1,2}$ However, such complications have not been reported with Pl-TB. We are reporting two cases of bilateral extensive lower limb DVT associated with tuberculosis (TB), one pleural and another lung parenchymal, occurring during anti-tubercular treatment (ATT) with daily isoniazid (H), rifampicin $(\mathrm{R})$, pyrazinamide $(\mathrm{Z})$ and ethambutol $(\mathrm{E})$.

\section{Case Report:}

\section{Case number one:}

A26-year-old male presented with low-grade fever, left sided pleuritic chest pain and heaviness of chest on left side. Patient had no addiction, totally ambulatory, and without any other present or past medical or surgical illness. His clinicoradiological findings were consistent with left sided pleural effusion. Aspirated pleural fluid was straw coloured, exudative, lymphocytic ( $95 \%$ of total cell count $7760 / \mathrm{cmm}$ ) with adenosine deaminase (ADA) $72 \mathrm{U} / \mathrm{L}$ (positive $>60 \mathrm{U} /$ L); Ziehl-Neelsen (Z-N) staining negative and mycobacterial tuberculosis (MTB) culture negative. Sputum for acid fast bacilli (AFB) smear for consecutive three days was negative and Mantoux test with 5 TU PPD showed $18 \times 12 \mathrm{~mm}$ indurations with blister formation. CT scan thorax with contrast revealed left sided pleural effusion without any lung parenchymal lesion. Closed percutaneous pleural biopsy by Abram's pleural biopsy needle from left side showed necrotizing epitheloid granuloma but MTB culture was negative from that specimen. Patient was diagnosed as tuberculous pleural effusion. He was put on ATT with daily H-300mg, R-600mg, Z-1500mg and E-1200mg. Patient tolerated the drugs well; he was ambulatory and clinically improved. On the 18th day of treatment he suddenly developed pain both lower limbs starting from the inguinal areas along with swelling of both lower limbs. A Doppler study done and showed extensive sub-acute thrombus with continuous involvement of deep venous system of both lower limbs up to external iliac veins [Fig.-1].

Further investigation revealed protein $\mathrm{C}$ antigenic assay levels were 115 per cent (normal value $70-140 \%$ ), protein $\mathrm{S}$ antigenic assay level was 72 per cent (normal value $70-123$ $\%$ ), bleeding time was 3 minutes, clotting time was 4 minutes and fibrin degradation product (FDP) was elevated with decreased antithrombin III level. Patient was treated with enoxaparin $80 \mathrm{mg}$ /day along with supportive measures, and

1. RMO cum Clinical Tutor, Department of Pulmonary Medicine NRS Medical College and Hospital, 138 AJC Bose Road, Shealdah, Kolkata-700014 West Bengal, India

2. Professor, Department of Pulmonary Medicine, NRS Medical College and Hospital, 138 AJC Bose Road, Shealdah, Kolkata-700014 West Bengal, India

3. RMO cum Clinical Tutor, Department of Pulmonary Medicine NRS Medical College and Hospital 138 AJC Bose Road, Shealdah,Kolkata-700014, West Bengal, India

4. Post Graduate Trainee Department of Pulmonary Medicine, NRS Medical College and Hospital,138 AJC Bose Road, Shealdah, Kolkata-700014 West Bengal, India

Corresponding author: Dr. Kaushik Saha, Rabindra Pally, e mail-doctorkaushiksaha@gmail.com 


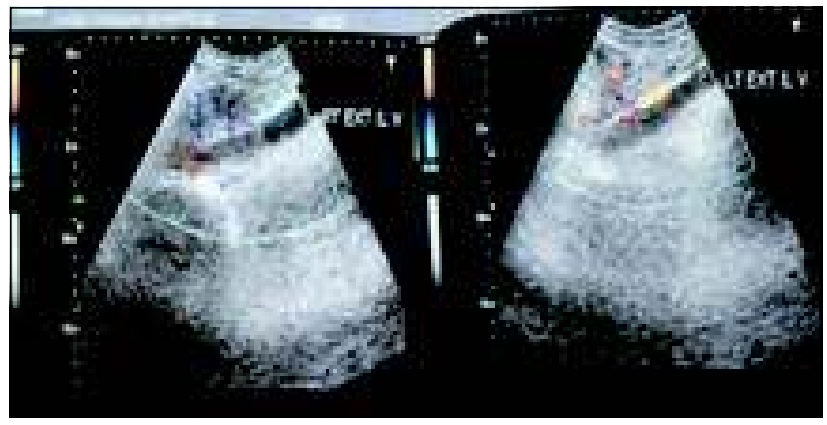

Fig-2: Colour doppler study of thigh showing involvement of both external iliac veins by extensive sub-acute thrombus.

switched on to warfarin (starting dose $5 \mathrm{mg}$ ) with maintenance of international normalized ratio (INR) between 2.5 to 3. Rifampicin was withdrawn and injection streptomycin $750 \mathrm{mg}$ daily was added in the regimen. A repeat Doppler study after three months of treatment with anticoagulant showed absence of thrombus with normal flowed phasic variation with inspiration and augmentation with distal compression (Fig.-2).

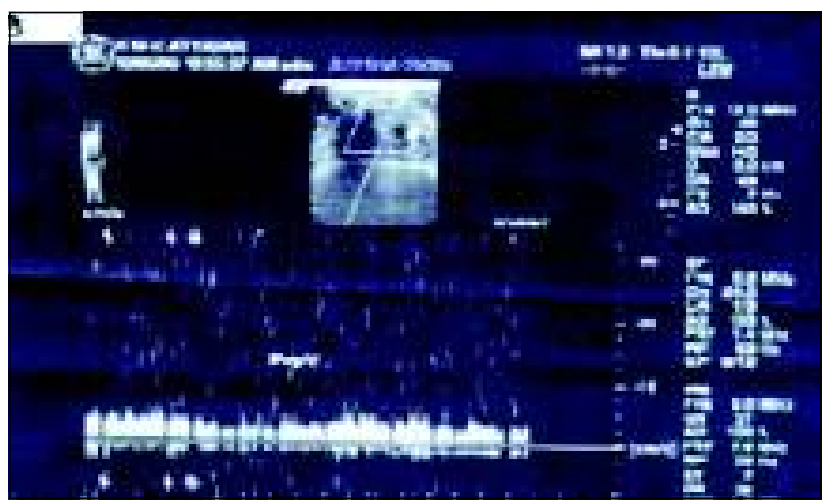

Fig-3: Colour doppler study of case number one in follow up showing disappearance of thrombus in the popliteal vein

Intensive phase of treatment was continued for first two months and continuation phase with isonizid and ethambutol for ten months. Warfarin was continued for 6 months. Rest of his treatment period was uneventful.

\section{Case number two:}

A 22-year-old man, non-smoker, non-diabetic, was presented with cough, mucoid expectoration and low grade fever. One of his three sputum AFB smear sample was positive and his $X$-ray chest showed infiltrative lesion in the right upper zone. He was diagnosed as smear $(+v e)$ P-TB. He was treated with daily H-300mg, R-450mg, Z-1500mg and E-800mg. He was ambulatory and without any risk factor for DVT. On the $2^{\text {nd }}$ week of treatment he complained of pain both lower limbs starting from the inguinal areas along with swelling. He was admitted for investigation. On the $2^{\text {nd }}$ day of admission he developed sudden onset of central chest pain, dyspnea with heart rate $120 / \mathrm{min}$, respiratory rate $36 / \mathrm{min}$ and blood pressure $60 / 40 \mathrm{~mm}$ of $\mathrm{Hg}$. He was diagnosed clinically as pulmonary thromboembolism. Treatment was started immediately with enoxaparin $80 \mathrm{mg} /$ day along with supportive measures, and switched on to warfarin (starting dose $5 \mathrm{mg}$ ) with maintenance of international normalized ratio (INR) between 2.5 to 3 . His HRCT thorax revealed typical sub pleural triangular opacity with base towards pleura (Fig.-3) suggestive of pulmonary infarction.

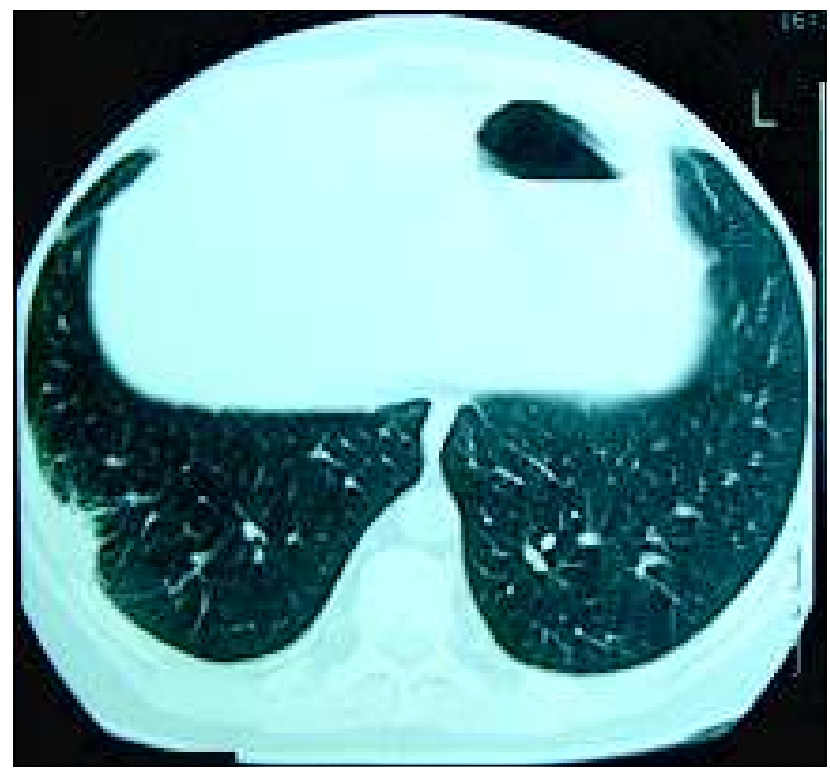

Fig-3: HRCT thorax of case number two showing sub pleural triangular opacity on right side, evidence of lung infarction.

A Doppler study showed extensive thrombus of the both lower limb extending up to external iliac veins of both sides and internal iliac vein of right side. Possibilities of occult carcinoma, intra-abdominal growth and coagulation defects were excluded. Rifampicin was stopped with addition of streptomycin and total duration of ATT was twelve months. He recovered completely and follow up Doppler study was normal after two months of treatment. Warfarin was continued for 6 months.

\section{Discussion:}

Pl-TB can occur singly or may be associated with PTB. Local complications of Pl-TB include empyema, pneumothorax, pyopneumothorax or mixed tuberculous empyema. ${ }^{3}$ One of the common sequelae of Pl-TB is residual pleural thickening, 
which occur as many as $50 \%$ of patients with TB pleurisy. ${ }^{4}$ However, systemic complications like vascular and hematological abnormalities are uncommon in PTB, more so in Pl-TB. Only few reports illustrated the associations between PTB and DIC. Kaminskaia GO et al. showed subtle changes in blood rheologic properties and haemostatic system in patients with PTB that included edema, rapid depletion, lower resistance and higher aggregation of erythrocyte, and increased haematocrit but normal erythrocyte count. ${ }^{1}$ They found an increase in activated partial thromboplastin time and thrombin time; a reduction in the values of the prothrombin indices and anti-thrombin III activity; normal or reduced levels of fibrinogen despite an increase in other acute phase reactants; and the appearance of large amounts of blocked fibrinogen in the blood. ${ }^{1}$ The analysis of the above findings enabled the authors to regard these changes as a sign of latent DIC syndrome. This hypothesis was clinically supported by an incidence of $3.2 \%$ of DIC with $63 \%$ mortality in culture proven tuberculosis. ${ }^{5}$ There were isolated reports of DIC due to anti-tubercular drugs, probably rifampicin. ${ }^{6}$ Studies also demonstrated a possible association between DVT and the use of rifampicin with a relative risk of 4.74 in patients treated with rifampicin containing regimens. ${ }^{7}$ This did not contraindicate the use of this drug in patients at risk, but such patients should be supervised.

It was also postulated that association between inflammation and haemostatic changes arising in PTB could result in hypercoagulable state which might predispose to deep vein thrombosis. Reactive thrombocytosis, elevations in plasma fibrinogen degradation products, tissue plasminogen activator and inhibitors, depressed anti thrombin III levels were demonstrated in active PTB, which worsen during first 2 weeks and normalized after a month of ATT. ${ }^{8}$ The risk of developing deep vein thrombosis was found to be increased with increased severity of PTB. ${ }^{8}$ Cases of deep vein thrombosis have been reported in patients with intraabdominal lymphadenopathy of tubercular etiology. ${ }^{2}$ High frequency of anti-phospholipid antibodies and deficiency of protein-S in patients with tuberculosis were mentioned in the literature. ${ }^{9}$ The possibility of undiagnosed pulmonary thromboembolism as a cause of sudden unexplained deaths in patients with tuberculosis was suggested.

In our first case, association between DVT and Pl-TB and in second case association between DVT and P-TB were established as both patients were young and ambulatory with no specific risk factors. In both of them underlying malignancy, intra-abdominal mass causing obstruction to the venous flow and congenital causes of hypercoagulability state were ruled out. Occurrence of DVT in the third week in the first case of small Pl-TB when effusion was improving both clinically and radiologically (in contrary to Bozoky G et al. finding) might suggest other than tubercular etiology for DVT. DVT resolution when rifampicin was withdrawn from the regime might indicate that the drug was the perpetrator. Those two cases highlighted the potential seriousness of this underreported phenomenon that might be associated with TB. We emphasized the need of awareness of DVT in TB patients, which could save precious lives.

\section{Conflict of Interest: None}

\section{References:}

1. Kaminskaia GO, Serebrianaia BA, Martynova EV, Mishin VI. Intravascular coagulation as a typical concomitant of acute pulmonary tuberculosis. Probl Tuberk 1997; 3: 42-6.

2. Gogna A, Pradhan GR, Sinha RSK, Gupta B. Tuberculosis presenting as deep vein thrombosis. Postgrad Med J 1999;75:104-106.

3. Chavalittamrong B, Angsusingha K, Tuchinda M. et al. Diagnostic significance of $\mathrm{pH}$, lactic acid dehydrogenase, lactate and glucose in pleural fluid. Respiration 1979; 38: 112-120.

4. Barbas CS, Cukier A, de Varvalho CR, et al. The relationship between pleural fluid findings and the development of pleural thickening in patients with pleural tuberculosis. Chest 1991; 100: 1264-1267.

5. Wang JY, Hsueh PR, Lee LN et al. Mycobacterium tuberculosis inducing disseminated intravascular coagulation. Thromb Haemost 2005; 93: 729-34.

6. Ip M, Cheng KP, Cheung WC. Disseminated intravascular coagulopathy associated with rifampicin. Tubercle 1991; 72: 291-3.

7. White NW. Venous thrombosis and rifampicin. Lancet 1989; 2: 1397.

8. Bozoky G, Ruby E, Goher I et al. Hematologic abnormalities in pulmonary tuberculosis. Orv Hetil 1997; 138: 1053-6.

9. Suarez Ortega S, Artiles Vizcaino J, Balda Aguirre I et al. Tuberculosis as risk factor for venous thrombosis. An Med Interna 1993; 10: 398-400. 